UAB-FT-364

hep-ph/9505287

\title{
Bound states of scalar bosons in extensions of the Standard Model
}

\author{
J. Cluab and J. A. Grifols \\ IFAE. Grup de Física Teòrica. \\ Universitat Autònoma de Barcelona \\ Bellaterra. Catalonia (Spain)
}

\begin{abstract}
We explore systematically, in a general two Higgs doublet model, the possibility that bound systems of scalar bosons do exist. We find a wide region of parameter space in the scalar potential for which S-wave bound states of Higgs bosons do indeed exist. On the contrary we show that the Minimal Supersimmetric Standard Model does not admit such bound systems.
\end{abstract}

\footnotetext{
${ }^{1}$ e-mail address:clua@ifae.es

${ }^{2}$ e-mail address:grifols@ifae.es
} 


\section{Introduction}

Progress in a deeper understanding of the Standard Model must probably follow from an elucidation of the rôle and inner workings of the Higgs boson sector in the theory. In this respect, of course, the experimental discovery of the Higgs scalar is crucial to the whole modern particle physics paradigm. This is therefore an extremely valuable experimental endevour that should be pursued in present and future machines. Still, on the theoretical side one would like to give answers to questions like: is the Higgs boson a fundamental particle, as quarks and leptons are or, else, is it a composite object? will its presence signal the existence of a new strong interacting regime of the weak interactions or, on the contrary will we get clues to deeper and most embrancing theoretical schemes, like Grand Unification or Supersymmetry?

Surely, the minimal standard model requires only one scalar boson doublet and it might well be that Nature makes use only of these minimal degrees of freedom. However, economy of Principles seems to be the guiding rule rather then economy of structures. Indeed, there is no present understanding for, e.g., the repetition of fermions of quarks and leptons. Furthermore, any extension of the standard gauge model paradigm that tries to encompass larger domains of reality is bound to contain a larger set of higgs scalar bosons. The Minimal Supersymmetric Standard Model (MSSM), for instance, requires two higgs boson doublets for it to be phenomenologically sound.

It seems therefore that scalar bosons or effective fields that, at the Fermi scale play the rôle of the Higgs boson degrees of freedom, are unavoidable in any sensible construction of a consistent paradigm of the fundamental physical laws. A rich phenomenology is therefore likely to develop involving the scalar sector of Physics Beyond The Standard Model.

In the present paper we explore systematically the possibility, first suggested in [1], that higgs bosons form bound states (Higgsonium). In [1] we established, setting up a particular two-higgs model, that non-relativistic loosely bound states of scalar bosons may indeed exist for a wide range of masses and couplings in the scalar potential. And this happens in a strictly perturbative regime, i.e. within the conventional Standard Model framework.

Section 2 is devoted to the discussion of the mathematical techniques used and the physical requirements needed for bound states to exist. In section 3 we present the extended higgs sectors and the corresponding lagrangians. An analysis of allowed regions in parameter space for bound states to appear follows in section 4 . Finally the last section (5) contains a summary of conclusions.

\section{Non relativistic bound states. The Yukawa Po- tential}

The Higgs potential in the standard model, or in any extension thereof, contains cubic and quartic scalar couplings. In the standard model these correspond to self-interactions of the higgs boson. In extended scalar potentials they do also involve interactions among different scalar bosons. In a two higgs model, for instance, with 5 physical fields (3 neutral, 2 charged) triple quartic couplings among charged and/or neutral scalars are present in the lagrangian ([2]). For certain domain of physical parameters, the triple boson terms can be interpreted as a classical density source of Yukawa field forces. As a result of these 
Yukawa interactions, binding among (heavy) scalars may result from exchange of a (light) scalar boson. This is the physical situation which we shall investigate in this paper.

The general physical requirements we must impose are

i) The Yukawa potential produces a non-relativistic (NR) bound state.

ii) The constituents of the bound states should outlive the bound-state annihilationtime.

iii) The period of revolution of the bound system should be shorter than both the lifetime of the constituents and the annihilation time of the system.

iv) The repulsive quartic interactions which may prevent binding are negligible.

If those requirements are met, then higgsonium is possible.

A generic triple scalar coupling can be written,

$$
\mathcal{L}_{3}=-\frac{\rho_{i j k}}{n !} H^{i} H^{j} H^{k}
$$

with $H^{i, j, k}$ being identical or different fields ( $\mathrm{n}$ is the number of identical fields). The Yukawa potential corresponding to the particles $i$ and $j$ exchanging particle $k$ is given by 3 ,

$$
V(r)=-V_{0} \frac{e^{-m_{k} r}}{m_{k} r},
$$

with

$$
V_{0}=\frac{\rho_{i i k} \rho_{j j k} M_{k}}{16 \pi M_{i} M_{j}}
$$

if particle $i$ is identical to particle $j$ then the potential is necessarily attractive.

The potential generated by the 4-coupling

$$
\mathcal{L}_{4}=\frac{\rho_{i j k l}}{n !} H^{i} H^{j} H^{k} H^{l}
$$

is

$$
V(\vec{r})=-\frac{\rho_{i i j j}}{4 M^{2}} \delta(\vec{r}) .
$$

This is a contact potential. We shall estimate its influence on higgsonium later.

If $V(r)$ is a Yukawa potential (or a sum of Yukawa potentials) then the corresponding Schrödinger equation

$$
\left(-\frac{d^{2}}{2 \mu d r^{2}}+\frac{l(l+1)}{2 \mu r^{2}}+V(r)\right) u_{n l}(r)=E_{n l} u_{n l}(r),
$$

with $\mu$ (the reduced mass) can be solved perturbatively by splitting the potential in a Coulombic piece (The Yukawa potential closely resembles a Coulomb potential near the origin) plus the deviation from an $1 / \mathrm{r}$ behaviour.

It is convenient to revert eq. (6) into an adimensional form by dividing by a mass parameter squared:

$$
\left(-\frac{d^{2}}{d x^{2}}+\frac{l(l+1)}{x^{2}}-\frac{2 g}{x} v(x)\right) \bar{u}_{n l}(x)=\mathcal{E}_{n l} \bar{u}_{n l}(x) .
$$


where the adimensional constant $2 \mathrm{~g}$ characterizes the strength of the potential. $\mathcal{E}_{n l}$ and $\bar{u}_{n l}(x)$ are the adimensional eigenvalues and eigenfunctions.

This equation is then split in an hydrogen problem with "hamiltonian"

$$
H_{0}=\left(-\frac{d^{2}}{d x^{2}}+\frac{l(l+1)}{x^{2}}-\frac{2 g}{x}\right)
$$

plus a perturbation

$$
\Delta H=-\frac{2 g}{x}(v(x)-1) .
$$

First order perturbation theory gives,

$$
\mathcal{E}_{n l}=-\frac{g^{2}}{n^{2}}+\langle n l|\Delta H| n l\rangle
$$

where $|n l\rangle$ are hydrogen-like wave functions.

Next step is to expand $\Delta H$ in a Taylor series in $x$

$$
\Delta H=-2 g \sum_{n=0}^{\infty} v_{n} x^{n}
$$

and realize that $\langle n l|\Delta H| n l\rangle$ can be written in terms of the expectation values

$$
\left\langle n l\left|r^{p}\right| n l\right\rangle=\langle p\rangle g^{-p}
$$

where

$$
\langle p\rangle=\frac{n^{p-1}}{2^{p+1}} \frac{(n-l-1) !}{(n+l) !} \int_{0}^{\infty} d x e^{-x} x^{2+2 l+p}\left(L_{n-l-1}^{2 l+1}(x)\right)^{2} .
$$

finally, then

$$
\mathcal{E}_{n l}=-g^{2}\left(n^{-2}+2 v_{0} g^{-1}+2 v_{1}\langle 1\rangle g^{-2}+2 v_{2}\langle 2\rangle g^{-3}\right)+O\left(1 / g^{2}\right) .
$$

For a single Yukawa potential,

$$
v_{n}=\frac{(-1)^{n+1}}{(n+1) !} \quad \text { and } \quad g=\frac{\mu V_{0}}{m^{2}} .
$$

This is an asymptotic series in $g^{-1}$ and gives accurate results for large enough $g$, i.e. for a sufficiently strong potential. However, for loosely bound systems like the ones we shall encounter, the convergence is improved notably ([4]) by the use of Padé approximants ([5]). We include in appendix A a short account on the method. It will permit us to establish a threshold condition (a value for the parameters of the potential) for a bound state to be formed (i.e. $\mathcal{E}_{n l} \leq 0$ ).

The basic issue here is to find a threshold value for $g$, i.e. a value above which the potential binds. From a numerical study close to this threshold, both for $l=0$ and $l=1$, one concludes that optimal Padé approximants for the energy are ${\sqrt{-\mathcal{E}_{10}}}^{[2 / 1]}$ and $-\mathcal{E}_{n l}^{[2 / 1]}$ for S-wave and $\mathrm{P}$-wave states respectively. The explicit forms for these Padé approximants are given in (50), using the coefficients of equation (14). As an example, in the case of single excange we obtain for the $l=0$ energy

$$
{\sqrt{-\mathcal{E}_{n 0}}}^{[2 / 1]}=\frac{g}{n} \frac{1+\frac{1-n^{2}}{3} \frac{1}{g}-\frac{n^{2}\left(4+5 n^{2}\right)}{12} \frac{1}{g^{2}}}{1+\frac{1}{3} \frac{1+2 n^{2}}{3} \frac{1}{g}}
$$


and the threshold coupling $g$,

$$
g_{n 0}^{[2 / 1]}=\frac{1}{6}\left(n^{2}-1+\sqrt{16 n^{4}+10 n^{2}+1}\right) .
$$

The self-consistency of the approach requires that we deal with non-relativistic systems, i.e. when binding is much smaller than rest-mass:

$$
E_{n l} \ll 2 M
$$

This is physically achieved for small strength, large range (light exchanged mass) potentials. As we shall explicitly show, these conditions can be actually met in 2 higgs models.

We realized before that a contact potential might effect our bound states. The perturbation induced on the $|n l\rangle$ states of higgsonium by a potential of the form

$$
-\frac{\rho_{i i i i}}{4 M^{2}} \delta(\vec{r})
$$

can be estimated to be

$$
\langle\text { Contact }\rangle \propto \rho_{i i i i} \frac{m^{3}}{4 M^{2}}
$$

for wavefunctions which correspond to potentials that are Coulombic near the origin, i.e.

$$
\Psi_{n l} \propto m^{3 / 2} r^{l} \quad \text { when } \quad r \rightarrow 0 .
$$

This is to be compared with

$$
\langle Y u k a w a\rangle \propto \frac{m^{2}}{M}
$$

The ratio

$$
\frac{\langle\text { Contact }\rangle}{\langle\text { Yukawa }\rangle} \propto \frac{m}{M}
$$

is small if we restrict ourselves to the nonrelativistic regime.

To comply with criteria ii) and iii) stated before it is necessary now to introduce a "characteristic time" for the bound state. We can give various definitions for this time, all differing only by a numerical factor of order one. Perhaps the most obvious thing to do is to define a "classical revolution period", i.e. the time it takes for the constituents to complete a "Bohr orbit":

$$
\tau=2 \pi \frac{\langle r\rangle}{\langle v\rangle}
$$

with

$$
\langle r\rangle_{n l}=\langle n l|r| n l\rangle \quad \text { and } \quad\langle v\rangle_{n l}=\frac{1}{m}\langle n l|p| n l\rangle
$$

But, a perfectly sensible alternative would be to use the inverse of the binding energy. The Heisenberg principle guarantees the physical consistency of these characteristic times.

With such a time, one can then apply criteria ii) and iii) to higgsonium. One merely requires that the width of the bound state and its constituents is smaller than the inverse characteristic time

\footnotetext{
${ }^{3}$ whenever required we used Hulthén [6] wavefunctions to obtain quantitative numerical estimates of equations such as (22), (24) or (25).
} 


\section{$3 \quad$ Extended Higgs boson sectors}

The most simple and natural extension of the Standard Model involves two complex higgs scalar $S U(2)_{L}$ doublets $\Phi_{1}$ and $\Phi_{2}$ with hyperchange $Y=1 / 2$.

The most general higgs potential -gauge invariant, $\mathrm{CP}$-conserving and renormalizablewith automatic exclusion of FCNC is,

$$
\begin{aligned}
& V\left(\phi_{1}, \phi_{2}\right)= \\
& \quad \mu_{1}^{2} \phi_{1}^{\dagger} \phi_{1}+\mu_{2}^{2} \phi_{2}^{\dagger} \phi_{2}+\lambda_{1}\left(\phi_{1}^{\dagger} \phi_{1}\right)^{2}+\lambda_{2}\left(\phi_{2}^{\dagger} \phi_{2}\right)^{2}+ \\
& \quad \lambda_{3}\left(\phi_{1}^{\dagger} \phi_{1}\right)\left(\phi_{2}^{\dagger} \phi_{2}\right)+\lambda_{4}\left(\phi_{1}^{\dagger} \phi_{2}\right)\left(\phi_{2}^{\dagger} \phi_{1}\right)+\lambda_{5}\left[\left(\phi_{1}^{\dagger} \phi_{2}\right)^{2}+\left(\phi_{2}^{\dagger} \phi_{1}\right)^{2}\right]
\end{aligned}
$$

where all constants are real.

In addition, this potential is bounded below if

$$
\lambda_{1}>0, \quad \lambda_{2}>0, \quad \lambda_{3}+\lambda_{4}+\lambda_{5}>-2 \sqrt{\lambda_{1} \lambda_{2}}, \quad \lambda_{4}+\lambda_{5}<0
$$

Spontaneous breaking of the electroweak symmetry down to electromagnetism occurs when $\phi_{1}$ and $\phi_{2}$ acquire v.e.v's $v_{1}$ and $v_{2}$ (which we take both real, since we are not interested in spontaneous CP violation). In terms of these vev's we rewrite the fields as,

$$
\phi_{1}=\frac{1}{\sqrt{2}}\left(\begin{array}{c}
\phi_{1}^{+} \\
v_{1}+R_{1}+i I_{1}
\end{array}\right) \quad \phi_{2}=\frac{1}{\sqrt{2}}\left(\begin{array}{c}
\phi_{2}^{+} \\
v_{2}+R_{2}+i I_{2}
\end{array}\right)
$$

where $R_{i}=\operatorname{Re} \phi_{i}^{0}-v_{i}$ and $I_{i}=\operatorname{Im} \phi_{i}^{0}$.

Also,

$$
\begin{aligned}
& \mu_{1}^{2}=-\lambda_{1} v_{1}^{2}-\frac{1}{2}\left(\lambda_{3}+\lambda_{4}+\lambda_{5}\right) v_{2}^{2}, \\
& \mu_{2}^{2}=-\lambda_{2} v_{2}^{2}-\frac{1}{2}\left(\lambda_{3}+\lambda_{4}+\lambda_{5}\right) v_{1}^{2} .
\end{aligned}
$$

The physical degrees of freedom, in terms of the 8 real fields in $\phi_{1}$ and $\phi_{2}$ are explicitly given by,

$$
H^{ \pm}=-\phi_{1}^{ \pm} \sin \beta+\phi_{2}^{ \pm} \cos \beta
$$

i.e. two charged higgs bosons, and

$$
A^{0}=-I_{1} \sin \beta+I_{2} \cos \beta
$$

a pseudoscalar neutral boson and, finally, the neutral scalars

$$
\begin{aligned}
H^{0} & =R_{1} \cos \alpha+R_{2} \sin \alpha \\
h^{0} & =-R_{1} \sin \alpha+R_{2} \cos \alpha,
\end{aligned}
$$

with

$$
\tan \beta=v_{2} / v_{1} \quad, \quad \tan 2 \alpha=\frac{C}{A-B}
$$




$$
A=v_{1}^{2} \lambda_{1} \quad, \quad B=v_{2}^{2} \lambda_{2} \quad, \quad C=v_{1} v_{2}\left(\lambda_{3}+\lambda_{4}+\lambda_{5}\right)
$$

Their masses are:

$$
\begin{aligned}
m_{H^{ \pm}}^{2} & =-\frac{1}{2}\left(v_{1}^{2}+v_{2}^{2}\right)\left(\lambda_{4}+\lambda_{5}\right) \\
m_{A^{0}}^{2} & =-\lambda_{5}\left(v_{1}^{2}+v_{2}^{2}\right) \\
m_{H^{0}, h^{0}}^{2} & =A+B \pm \sqrt{(A-B)^{2}+C^{2}}
\end{aligned}
$$

For convenience of analysis, in section 4 , we shall trade the 7 parameters $\mu_{1}, \mu_{2}, \lambda_{1,2,3,4,5}$ for the 7 related ones: the masses $m_{H^{ \pm}}, m_{A^{0}}, m_{H^{0}}, m_{h^{0}}$, the mixing angles $\alpha$ and $\beta$, and the vev $v=\sqrt{\frac{1}{2}\left(v_{1}^{2}+v_{2}^{2}\right)}$.

Finally, to specify completely our model, we take doublet $\phi_{1}$ to be coupled to all down-type right-handed $(\mathrm{RH})$ fermions and hence gives masses to $d, s, b$ quarks and to the charged leptons $(e, \mu, \tau)$. Similarly, the higgs field $\phi_{2}$ is coupled to up-type $(u, c, t)$ $\mathrm{RH}$ fermion fields and is responsible for their masses.

The neutral higgs bosons will have to be more massive than $60 \mathrm{GeV}$. The charged higgs boson masses should not lie below $45 \mathrm{GeV}$.

As to $\tan \beta$, we will require

$$
0.7<\tan \beta<50
$$

This comes about from the requirement that the higgs boson couplings to the fermions are perturbative (of course, the actual restrictions arise from the top and bottom quarks).

\section{Results}

The states that can be a priori constructed (the forces being necessarily attractive) are $\left\langle H^{+} H^{-}\right\rangle,\left\langle A^{0} A^{0}\right\rangle,\left\langle h^{0} h^{0}\right\rangle$ and $\left\langle H^{0} H^{0}\right\rangle$. However, $\left\langle h^{0} h^{0}\right\rangle$ and $\left\langle H^{0} H^{0}\right\rangle$ would not qualify as non-relativistic systems since $m_{H^{0}}>m_{h^{0}}$ and $H^{0}$ is the particle to be exchanged. We therefore shall study the S-wave states $\left\langle H^{+} H^{-}\right\rangle_{10}$ and $\left\langle A^{0} A^{0}\right\rangle_{10}$.

We have analyzed also the $\mathrm{P}$-wave states, but due to the centrifugal barrier their formation is not possible.

\subsection{The state $\left\langle H^{+} H^{-}\right\rangle_{10}$}

This state can be formed by the exchange of $H^{0}$ and $h^{0}$ (the vertex $H^{+} H^{-} A^{0}$ does not exist) whose Feynman rules are collected in appendix B.

To cope with a 6 -parameter analysis we shall in what follows choose definitive sets of masses and plot in the $(\alpha, \tan \beta)$-plane the corresponding allowed regions where the state $\left\langle H^{+} H^{-}\right\rangle$is possible, i.e. where $\mathcal{E}_{10}<0$ and criteria $\left.i\right)$, ii) and iii) are fullfilled.

The choice of masses is dictated by the requirements of producing non-relativistic systems (constituent mass much larger than exchanged mass), exceeding the lower bounds of $45 \mathrm{GeV}$ and $60 \mathrm{GeV}$ for charged and neutral scalars respectively, and complying with unitarity (i.e. scalar masses below about $1000 \mathrm{GeV}$ ).

In order to compare the "orbiting time" with the lifetime of the constituents $H^{ \pm}$we need to compute the decays of the charged higgs boson. Appendix $\mathrm{C}$ is a full listing of the partial widths of $\left(H^{ \pm}, H^{0}, A^{0}, h^{0}\right)$. From these formulae we easily calculate the relevant $H^{ \pm}$total width. 
The lifetime of the $\left\langle H^{+} H^{-}\right\rangle_{10}$ state is obtained [7] from the width,

$$
\Gamma\left(\left\langle H^{+} H^{-}\right\rangle_{10}\right)=|\Psi(0)|^{2} v_{r e l} \sigma\left(H^{+} H^{-} \rightarrow \text { all }\right)
$$

where

$$
\sigma\left(H^{+} H^{-} \rightarrow a l l\right)=\sigma\left(H^{+} H^{-} \rightarrow H^{i} H^{j}, t \bar{t}, W^{+} W^{-}, Z Z, \cdots\right)
$$

For the wave function at the origin we use the Hulthén wave function.

In figures (11), (2) and (3) we display our numerical results, for the constituent masses of $300 \mathrm{GeV}$, $400 \mathrm{GeV}$ and $500 \mathrm{GeV}$, respectively, and the neutral higgs boson masses shown. We see clearly that there is much parameter space to allow for higgsonium.

\subsection{The state $\left\langle A^{0} A^{0}\right\rangle_{10}$}

It can be formed via the exchange of $H^{0}$ and $h^{0}$ bosons. The corresponding couplings are explicitly given by eqs. B.7 and B.8.

In our analysis we shall demand that $m_{A^{0}}$ is much larger than $m_{H^{0}}$ and $m_{h^{0}}$ (recall the non-relativistic character of the approach).

The construction of the allowed regions in parameter space follows the same strategy as for the $\left\langle H^{+} H^{-}\right\rangle_{10}$-states.

The results are shown in figures (4) and (5) ( for $m_{A^{0}}=400 \mathrm{GeV}$ and $500 \mathrm{GeV}$, respectively).

Again, formation of higgsonium $\left\langle A^{0} A^{0}\right\rangle_{10}$ is possible, at least for a considerable range of higgs boson masses and mixing angles.

\subsection{The MSSM case}

In the MSSM ( 8 ), the extra constraints imposed by supersymmetry on the scalar potential lead to the following couplings:

$$
\begin{aligned}
\rho_{A^{0} A^{0} H^{0}}^{M S S M} & =\frac{i g m_{Z}}{2 \cos \theta_{W}} \cos 2 \beta \cos (\beta+\alpha) \\
\rho_{A^{0} A^{0} h^{0}}^{M S S M} & =\frac{i g m_{Z}}{2 \cos \theta_{W}} \cos 2 \beta \sin (\beta+\alpha) \\
\rho_{H^{+} H^{-} H^{0}}^{M S S M} & =-i g\left(m_{W} \cos (\alpha-\beta)-\frac{m_{Z}}{2 \cos \theta_{W}} \cos 2 \beta \cos (\alpha+\beta)\right) \\
\rho_{H^{+} H^{-} H^{0}}^{M S S M} & =-i g\left(m_{W} \sin (\alpha-\beta)+\frac{m_{Z}}{2 \cos \theta_{W}} \cos 2 \beta \sin (\alpha+\beta)\right) .
\end{aligned}
$$

These couplings should be responsible for the formation of the $\left\langle H^{+} H^{-}\right\rangle_{10}$ and $\left\langle A^{0} A^{0}\right\rangle_{10}$ bound states. However, using the techniques explained before, we reach the conclusion thath their strength is not sufficient for these bound states to exist. Therefore, the MSSM does not produce higgsonium.

\section{Summary}

The Higgs boson is the only element of the Standard Model yet to be disclosed by experiment. In the minimal version of the standard electroweak model, only one neutral Higgs 
scalar exists, but non minimal extensions are possible and even theoretically desirable. Indeed more ambitious constructs such as Grand Unified Theories or Supersymmetry demand larger scalar sectors. The most natural extension of the minimal scalar sector is to include two $S U(2)_{L}$ doublets. This extension implies the existence of two charged scalars particles and three neutral particles.

In this paper we have considered a generic two higgs doublet model and explored the possibilities that, for certain choices of the parameters in the scalar potential, bound states of Higgs scalars are produced. This should come about through the appearence of attractive Yukawa-type forces among identical Higgs particles, charged or neutral. We have shown that bound states of the systems $H^{+} H^{-}$and $A^{0} A^{0}$ in an S-wave mode are indeed possible for a wide range of parameters in the Higgs potential. Also, P-wave or higher angular momentum states are ruled out in 2-Higgs models. Since the Minimal Supersymmetric Standard Model is, as far as its Higgs sector is concerned, a model in the generic class of two Higgs models one might wonder whether scalar bound states can be achieved in this case. It turns out that this is not the case and the reason lies in the strong extra constraints imposed by Supersymmetry. In this case, the 6 parameter space $\left(m_{H^{ \pm}}, m_{H^{0}}, m_{h^{0}}, m_{A^{0}}, \alpha\right.$, and $\left.\tan \beta\right)$ reduces to a 2 parameter space: $\tan \beta$ and one scalar particle mass (e.g. $\left.m_{A^{0}}\right)$.

This work has been partially supported by CICYT under project under AEN-93-0474 


\section{A The Padé approximants}

Let $A(x)$ be a function whose formal series expansion is

$$
A(x)=\sum_{j=0}^{\infty} a_{j} x^{j}
$$

We define

$$
A^{[L / M]}(x)=\frac{P_{L}(x)}{Q_{M}(x)}
$$

as the $[L / M]$ Padé approximant of function $A(x)$, where $P_{L}(x)$ is a polynomial of order less or equal than $L$ and $Q_{M}(x)$ is a polynomial of order less or equal than $M . P_{L}(x)$ and $Q_{M}(x)$ verify:

1. $\sum_{j=0}^{\infty} a_{j} x^{j}-A^{[L / M]}(x)=O\left(x^{L+M+1}\right)$.

2. $Q_{M}(0)=1$.

3. $P_{L}$ and $Q_{M}$ do not have common factors.

Theorem 1 The Padé approximant $[L / M]$ to a formal series expansion (if it exists) is unique.

This theorem allows the explicit construction of the various approximants:

$$
\begin{aligned}
& {[0 / 0]=a_{0}} \\
& {[1 / 0]=a_{0}+a_{1} x} \\
& {[0 / 1]=\frac{a_{0}}{\left(a_{1} / a_{0}\right) x}} \\
& {[1 / 1]=\frac{a_{0}+\left(a_{1}-a_{0} a_{2} / a_{1}\right) x}{1-\left(a_{2} / a_{1}\right) x}} \\
& {[2 / 0]=a_{0}+a_{1} x+a_{2} x^{2}} \\
& {[2 / 1]=\frac{a_{0}+\left(a_{1}-a_{0} a_{3} / a_{2}\right) x+\left(a_{2}-a_{1} a_{3} / a_{2}\right) x^{2}}{1-\left(a_{3} / a_{2}\right) x}}
\end{aligned}
$$

Conjecture 1 (Padé) If a function $F(z)$ is regular inside a circle $|z|<R$ except for $m$ poles within this circle, then there exist at least a subsequence of the diagonal Padé approximants which converges uniformly to $F(z)$ inside the domain obtained by removing from this circle the interior of small circles centered at the poles.

This conjecture allows to obtain large convergence regions for large classes of functions. In practice one can test it on numerical examples. 


\section{B Feynman rules involving the Higgs bosons}

\section{B.1 Gauge bosons and Higgs bosons}

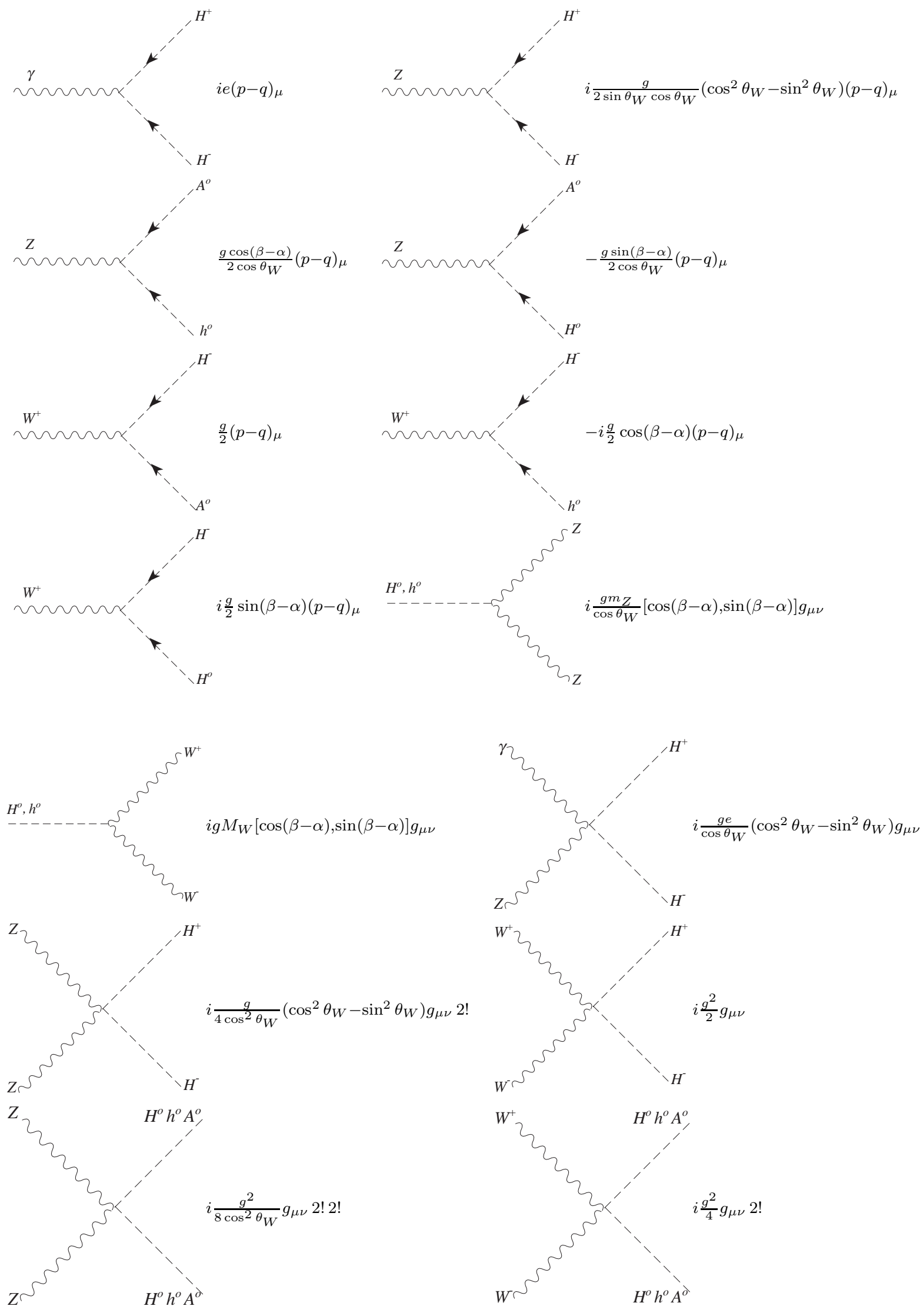




\section{B.2 Fermions and Higgs bosons}

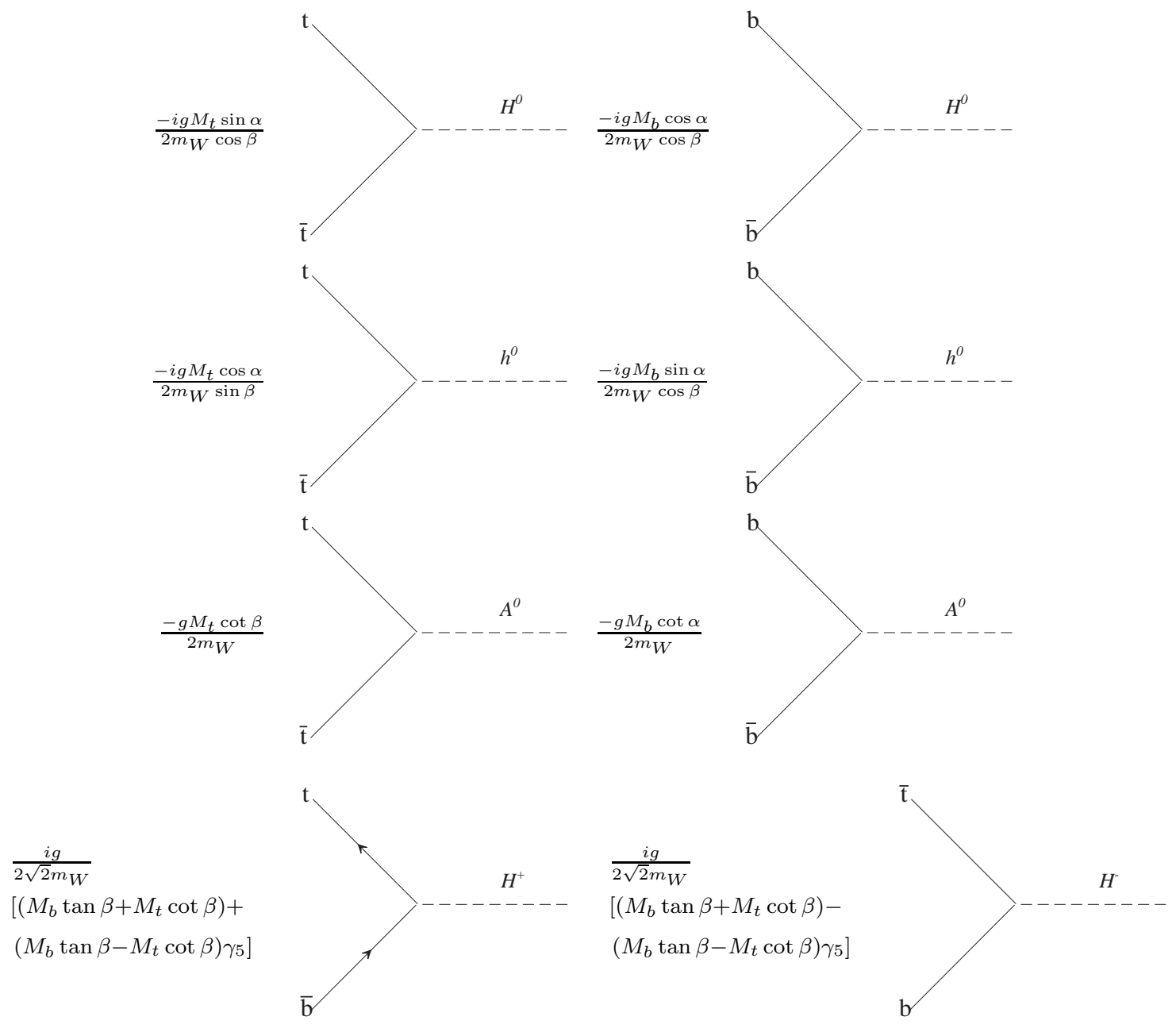

\section{B.3 3-scalar vertices}

$$
\begin{aligned}
& \rho_{H^{+} H^{-} H^{0}}=\frac{\csc \beta \sec \beta}{2^{5 / 2} v} \quad \begin{array}{l}
\left(2 m_{H^{ \pm}}^{2}(\sin (\alpha+\beta)-\sin (\alpha-3 \beta))\right)+ \\
\left(m_{H^{0}}^{2}(3 \sin (\alpha+\beta)+\sin (\alpha-3 \beta))\right)
\end{array} \\
& \left(m_{H^{0}}^{2}(3 \sin (\alpha+\beta)+\sin (\alpha-3 \beta))\right) \\
& \rho_{H^{+} H^{-} h^{0}}=\frac{\csc \beta \sec \beta}{2^{5 / 2} v}\left(2 m_{H^{ \pm}}^{2}(\cos (\alpha+\beta)-\cos (\alpha-3 \beta))\right)+ \\
& \left(m_{h^{0}}^{2}(3 \cos (\alpha+\beta)+\cos (\alpha-3 \beta))\right) \\
& \rho_{H^{0} H^{0} H^{0}}=\frac{\csc \beta \sec \beta}{2^{7 / 2} v} m_{H^{0}}^{2}(3 \sin (\alpha+\beta)-\sin (3 \alpha+\beta)) \\
& \rho_{h^{0} h^{0} h^{0}}=\frac{\csc \beta \sec \beta}{2^{7 / 2} v} m_{h^{0}}^{2}(3 \cos (\alpha+\beta)+\cos (3 \alpha+\beta)) \\
& \rho_{H^{0} H^{0} h^{0}}=\frac{\csc \beta \sec \beta}{2^{7 / 2} v}\left(2 m_{H^{0}}^{2}+m_{h^{0}}^{2}\right)(\sin 2 \alpha \sin (\alpha-\beta))
\end{aligned}
$$




$$
\begin{aligned}
& \rho_{h^{0} H^{0} H^{0}}=\frac{\csc \beta \sec \beta}{2^{7 / 2} v}\left(2 m_{h^{0}}^{2}+m_{H^{0}}^{2}\right)(\sin 2 \alpha \cos (\alpha-\beta)) \\
& \rho_{A^{0} A^{0} H^{0}}=\frac{\csc \beta \sec \beta}{2^{5 / 2} v} \quad\left(2 m_{A^{0}}^{2}(\sin (\alpha+\beta)-\sin (\alpha-3 \beta))\right)+ \\
& \rho_{A^{0} A^{0} h^{0}}=\frac{\csc \beta \sec \beta}{2^{5 / 2} v} \quad \begin{array}{l}
\left(2 m_{A^{0}}^{2}(\cos (\alpha+\beta)-\cos (\alpha-3 \beta))\right)+ \\
\left(m_{h^{0}}^{2}(3 \cos (\alpha+\beta)+\cos (\alpha-3 \beta))\right)
\end{array}
\end{aligned}
$$

\section{B.4 4-scalar vertices}

$$
\begin{aligned}
\rho_{H^{+} H^{-} H^{+} H^{-}}= & \frac{\csc ^{2} \beta \sec ^{2} \beta}{64 v^{2}}\left(\begin{array}{c}
m_{H^{0}}^{2}(\sin (\alpha-3 \beta)+3 \sin (\alpha+\beta))^{2}+ \\
m_{h^{0}}^{2}(\cos (\alpha-3 \beta)+3 \cos (\alpha+\beta))^{2}
\end{array}\right) \\
\rho_{H^{0} H^{0} H^{0} H^{0}}= & \frac{\csc ^{2} \beta \sec ^{2} \beta}{256 v^{2}}\left(\begin{array}{c}
m_{H^{0}}^{2}(\sin (3 \alpha-\beta)-3 \sin (\alpha+\beta))^{2}+ \\
\left.m_{h^{0}}^{2}(2 \sin (2 \alpha) \sin (\alpha-\beta))^{2}\right)
\end{array}\right) \\
\rho_{A^{0} A^{0} A^{0} A^{0}}=\frac{\csc ^{2} \beta \sec ^{2} \beta}{256 v^{2}} & \left.\begin{array}{c}
m_{H^{0}}^{2}(\sin (\alpha-3 \beta)+3 \sin (\alpha+\beta))^{2}+ \\
m_{h^{0}}^{2}(\cos (\alpha-3 \beta)+3 \cos (\alpha+\beta))^{2}
\end{array}\right) \\
\rho_{h^{0} h^{0} h^{0} h^{0}}=\frac{\csc ^{2} \beta \sec ^{2} \beta}{256 v^{2}} & \left(\begin{array}{l}
m_{H^{0}}^{2}(2 \sin (2 \alpha) \cos (\alpha-\beta))^{2}+ \\
m_{h^{0}}^{2}(\cos (3 \alpha-\beta)+3 \cos (\alpha+\beta))^{2}
\end{array}\right)
\end{aligned}
$$

Notice that vertices with an odd number of $A^{0}$ 's vanish (CP conservation)

\section{Partial widths of Higgs bosons}

\section{C.1 Fermionic decays}

To be definite, we display our formulae in terms of the 3rd family of quarks.

$$
\begin{aligned}
\Gamma\left(H^{+} \rightarrow t \bar{b}\right)= & \frac{3 g^{2} \lambda^{1 / 2}}{32 \pi m_{W}^{2} M_{H^{+}}^{3}}\left(\left(M_{H^{+}}^{2}-M_{b}^{2}-M_{t}^{2}\right)\right. \\
& \left.\left(M_{b}^{2} \tan ^{2} \beta+M_{t}^{2} \cot ^{2} \beta\right)-4 M_{b}^{2} M_{t}^{2}\right) \\
\Gamma\left(H^{i} \rightarrow t \bar{t}\right)= & \frac{3 g^{2} M_{t}^{2} u_{i}^{2} M_{i}}{32 \pi m_{W}^{2} \sin ^{2} \beta}\left(1-\frac{4 M_{t}^{2}}{M_{i}^{2}}\right)^{p}
\end{aligned}
$$




$$
\Gamma\left(H^{i} \rightarrow b \bar{b}\right)=\frac{3 g^{2} M_{b}^{2} d_{i}^{2} M_{i}}{32 \pi m_{W}^{2} \cos ^{2} \beta}\left(1-\frac{4 M_{t}^{2}}{M_{i}^{2}}\right)^{p}
$$

where

$$
u_{i} \equiv\left\{\begin{array}{rl}
-\sin \alpha, \quad H^{i} & =H^{0} \\
\cos \alpha, \quad H^{i} & =h^{0} \\
\cos \beta, & H^{i}=A^{0}
\end{array},\right.
$$

and

$$
d_{i} \equiv\left\{\begin{array}{rl}
\cos \alpha, \quad H^{i} & =H^{0} \\
\sin \alpha, \quad H^{i} & =h^{0} \\
-\sin \beta, & H^{i}=A^{0}
\end{array} .\right.
$$

Also

$$
p \equiv\left\{\begin{array}{ll}
3 / 2, & H^{i}=H^{0}, h^{0} \\
1 / 2, & H^{i}=A^{0}
\end{array} .\right.
$$

and

$$
\lambda^{1 / 2} \equiv\left[\left(M_{1}^{2}+M_{2}^{2}-M_{3}^{2}\right)^{2}-4 M_{1}^{2} M_{2}^{2}\right]^{1 / 2},
$$

( $M_{1} M_{2}$ i $M_{3}$ are the masses of the particles participating in the decay).

For decays into leptons, one should suppress the color factor 3.

\section{C.2 Decays into a pair of gauge bosons}

$$
\begin{aligned}
\Gamma\left(H^{i} \rightarrow W^{+} W^{-}\right) & =\frac{g^{2}\left(M_{H^{i}}^{4}-4 M_{H^{i}}^{2} m_{W}^{2}+12 m_{W}^{4}\right) V^{i}}{64 \pi m_{W}^{2} M_{H^{i}}}\left(1-\frac{4 m_{W}^{2}}{M_{H^{i}}^{2}}\right)^{1 / 2} \\
\Gamma\left(H^{i} \rightarrow Z Z\right) & =\frac{g^{2}\left(M_{H^{i}}^{4}-4 M_{H^{i}}^{2} m_{Z}^{2}+12 m_{Z}^{4}\right) V^{i}}{128 \pi m_{Z}^{2} \cos ^{2} \theta_{W} M_{H^{i}}}\left(1-\frac{4 m_{W}^{2}}{M_{H^{i}}^{2}}\right)^{1 / 2}
\end{aligned}
$$

with

$$
V^{i} \equiv\left\{\begin{array}{cl}
\cos ^{2} \alpha, & H^{i}=H^{0} \\
\sin ^{2} \alpha, & H^{i}=h^{0} \\
0, & H^{i}=A^{0}
\end{array}\right.
$$

\section{C.3 Decays into a gauge boson and a Higgs boson}

$$
\Gamma\left(H^{ \pm} \rightarrow W^{ \pm} H^{i}\right)=\frac{g^{2} \lambda^{1 / 2} G^{i}}{64 \pi m_{H^{ \pm}}^{3}}\left[m_{W}^{2}-2\left(m_{H^{ \pm}}^{2}+M_{H^{i}}^{2}\right)+\frac{\left(m_{H^{ \pm}}^{2}-M_{H^{i}}^{2}\right)^{2}}{m_{W}^{2}}\right],
$$

where

$$
\begin{gathered}
G^{i} \equiv\left\{\begin{array}{cc}
\sin ^{2} \alpha, & H^{i}=H^{0} \\
\cos ^{2} \alpha, & H^{i}=h^{0} \\
1, & H^{i}=A^{0}
\end{array} .\right. \\
\Gamma\left(H^{i} \rightarrow Z H^{j}\right)=\frac{g^{2} \lambda^{1 / 2} G^{i j}}{64 \pi M_{H^{i}}^{3}}\left[m_{Z}^{2}-2\left(M_{H^{i}}^{2}+M_{H^{j}}^{2}\right)+\frac{\left(M_{H^{i}}^{2}-M_{H^{j}}^{2}\right)^{2}}{m_{Z}^{2}}\right],
\end{gathered}
$$


where

$$
G^{i j} \equiv\left\{\begin{array}{cl}
\sin ^{2} \alpha, & H^{i}, H^{j}=H^{0}, A^{0} \\
\cos ^{2} \alpha, & H^{i}, H^{j}=h^{0}, A^{0} \\
0, & H^{i}, H^{j}=H^{0}, h^{0}
\end{array} .\right.
$$

\section{C.4 Decays into two Higgs scalars}

Whenever kinematically possible:

$$
\begin{aligned}
\Gamma\left(H^{i} \rightarrow H^{j} H^{k}\right)= & \frac{1}{4 \pi M_{H^{i}}}\left(1-\left(M_{H^{j}}+M_{H^{k}}\right)^{2} /\left(4 M_{H^{i}}^{2}\right)\right)^{1 / 2} \\
& \left(1-\left(M_{H^{j}}-M_{H^{k}}\right)^{2} /\left(4 M_{H^{i}}^{2}\right)\right)^{1 / 2} \rho_{i j k} \frac{1}{\left(1+\delta_{j k}\right) !},
\end{aligned}
$$

where $\rho_{i j k}$ is given by equations (51)-(58).

\section{References}

[1] J.A. Grifols, Phys. Lett. B264 (1991) 149; For related work see also L. Di Leo and J.W.Darewych, Phys. Rev. D49 (1994) 1659.

[2] J.F. Gunion, H.E. Haber, G. Kane and S. Dawson, The Higgs Hunter's Guide, Addison-Wesley (1990).

[3] J. J. Sakurai, Advanced Quantum Mechanics, Addison-Wesley (1967).

[4] A. Galindo and P. Pascual, Nuovo Cimento 30 A (1975) 111.

[5] G.A. Baker, Essentials of Padé approximants, Academic Press (1975); G.A. Baker and P. Graves-Morris, Padé approximants, in Encycl. of math. and its appl., Addison-Wesley Vol. 13, 14

[6] L. Hulthén and M. Sugawara, Handbuch der Physik Vol XXXIX (1957) 1.

[7] J. D. Jackson, Lectures on the new particles.

Proceedings of SLAC Summer Institute (1976) 147

[8] H. Haber and G. Kane, Phys. Rep. 117 (1985) 75. 

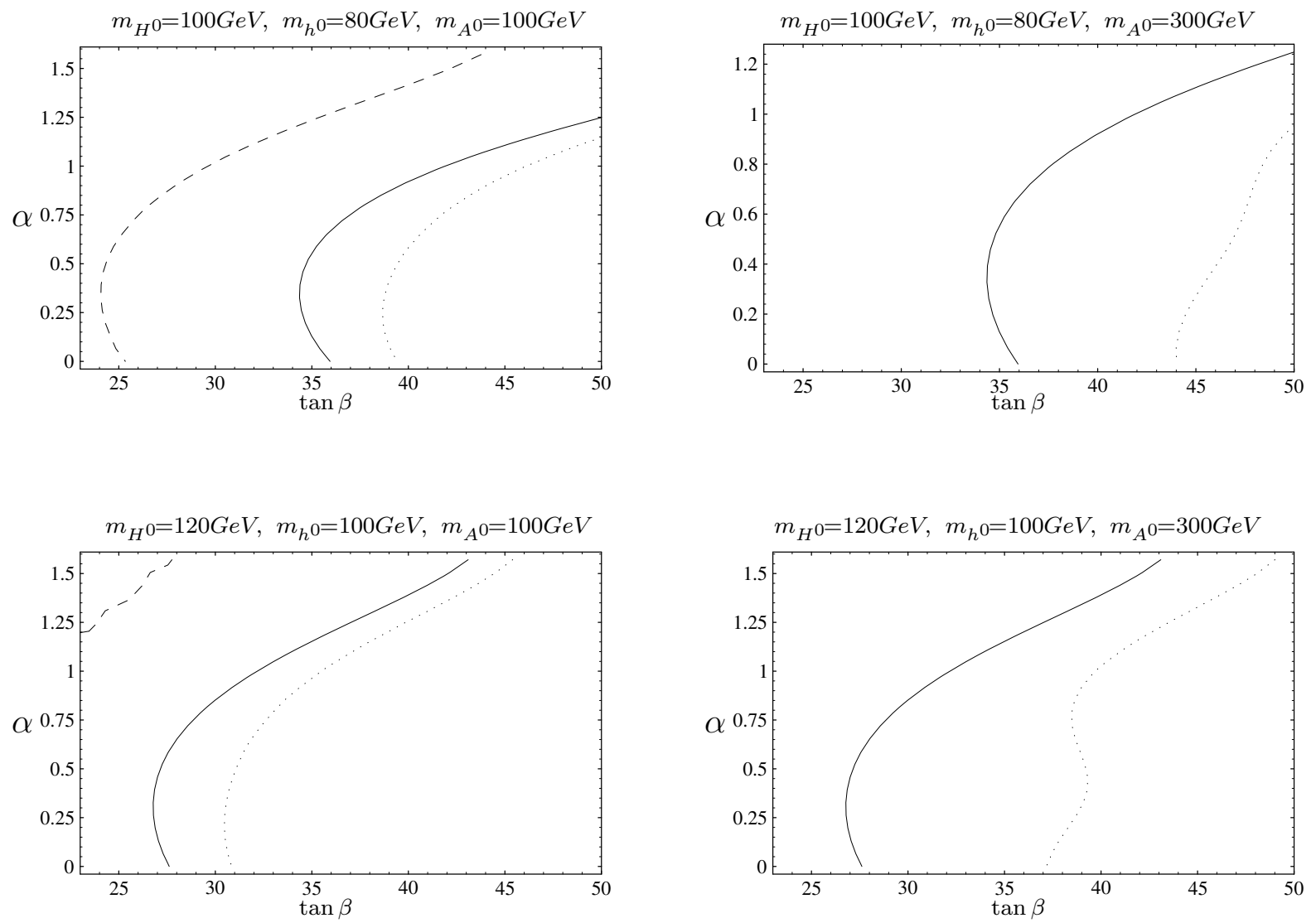

Figure 1: $\quad$ Allowed (shaded) areas in the $\alpha$ and $\tan \beta$ plane for $m_{H^{ \pm}}=300 \mathrm{GeV}$ and various choices of neutral Higgs boson masses. 

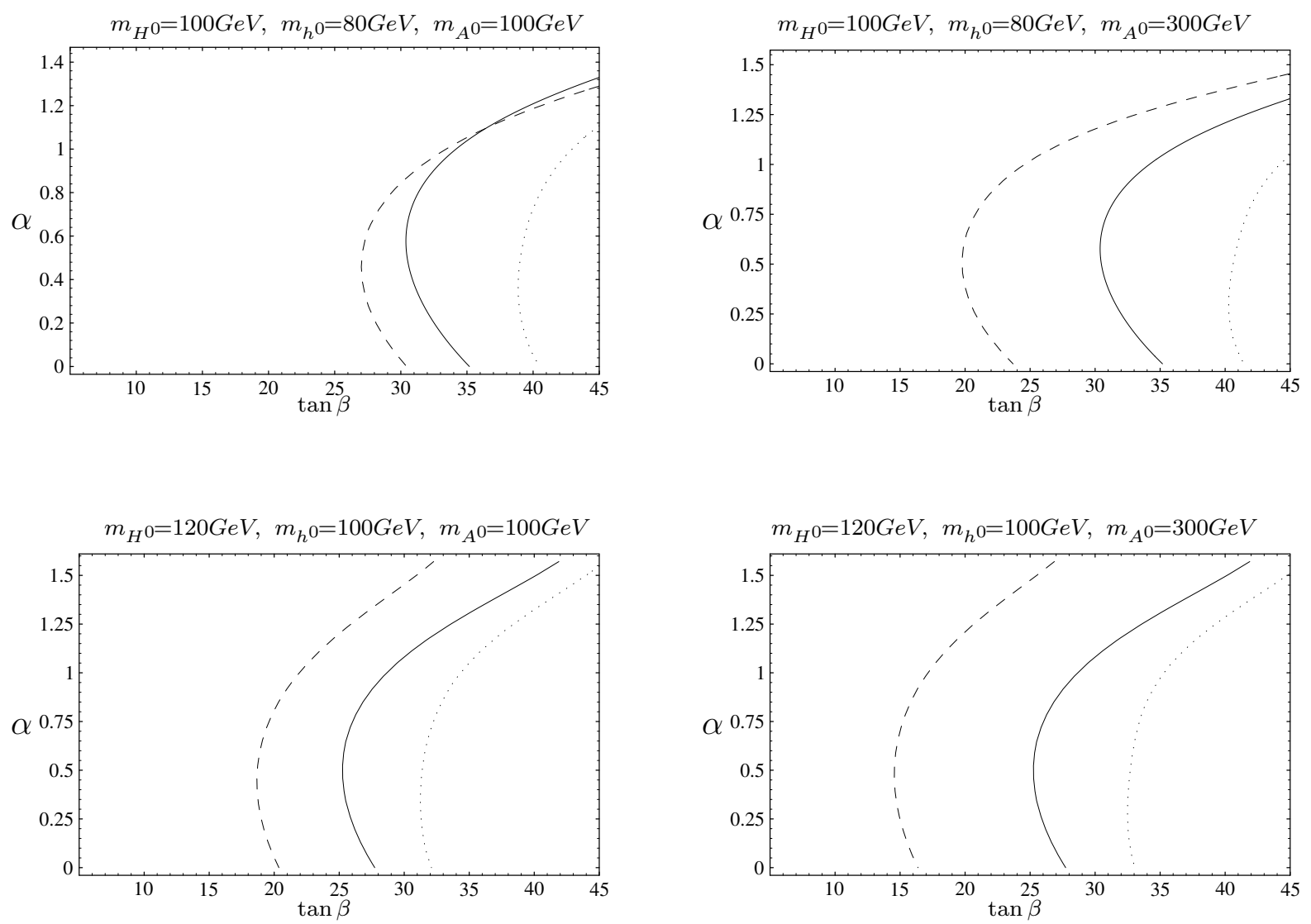

Figure 2: $\quad$ Allowed (shaded) areas in the $\alpha$ and $\tan \beta$ plane for $m_{H^{ \pm}}=400 \mathrm{GeV}$ and various choices of neutral Higgs boson masses. 

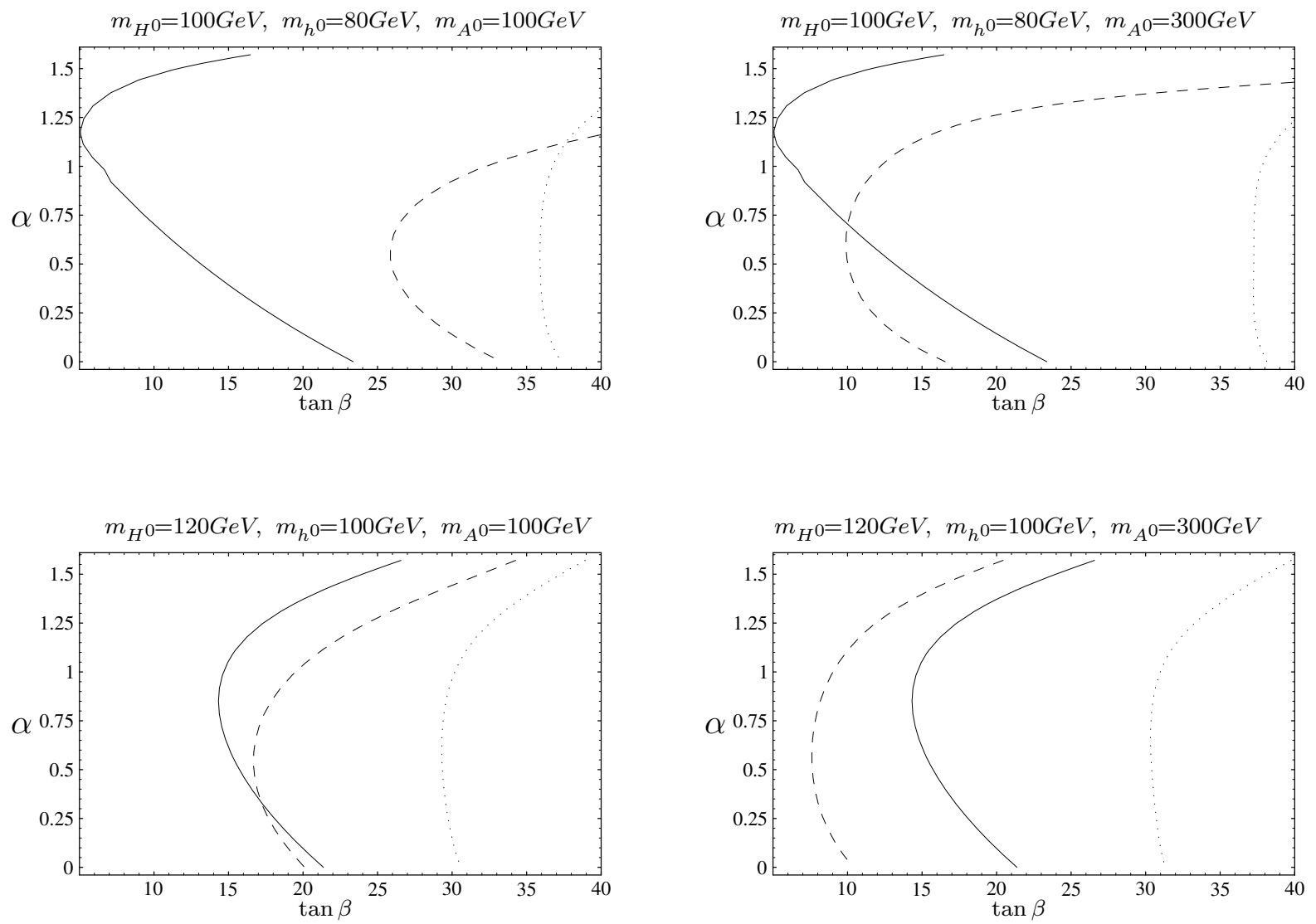

Figure 3: $\quad$ Allowed (shaded) areas in the $\alpha$ and $\tan \beta$ plane for $m_{H^{ \pm}}=500 \mathrm{GeV}$ and various choices of neutral Higgs boson masses. 

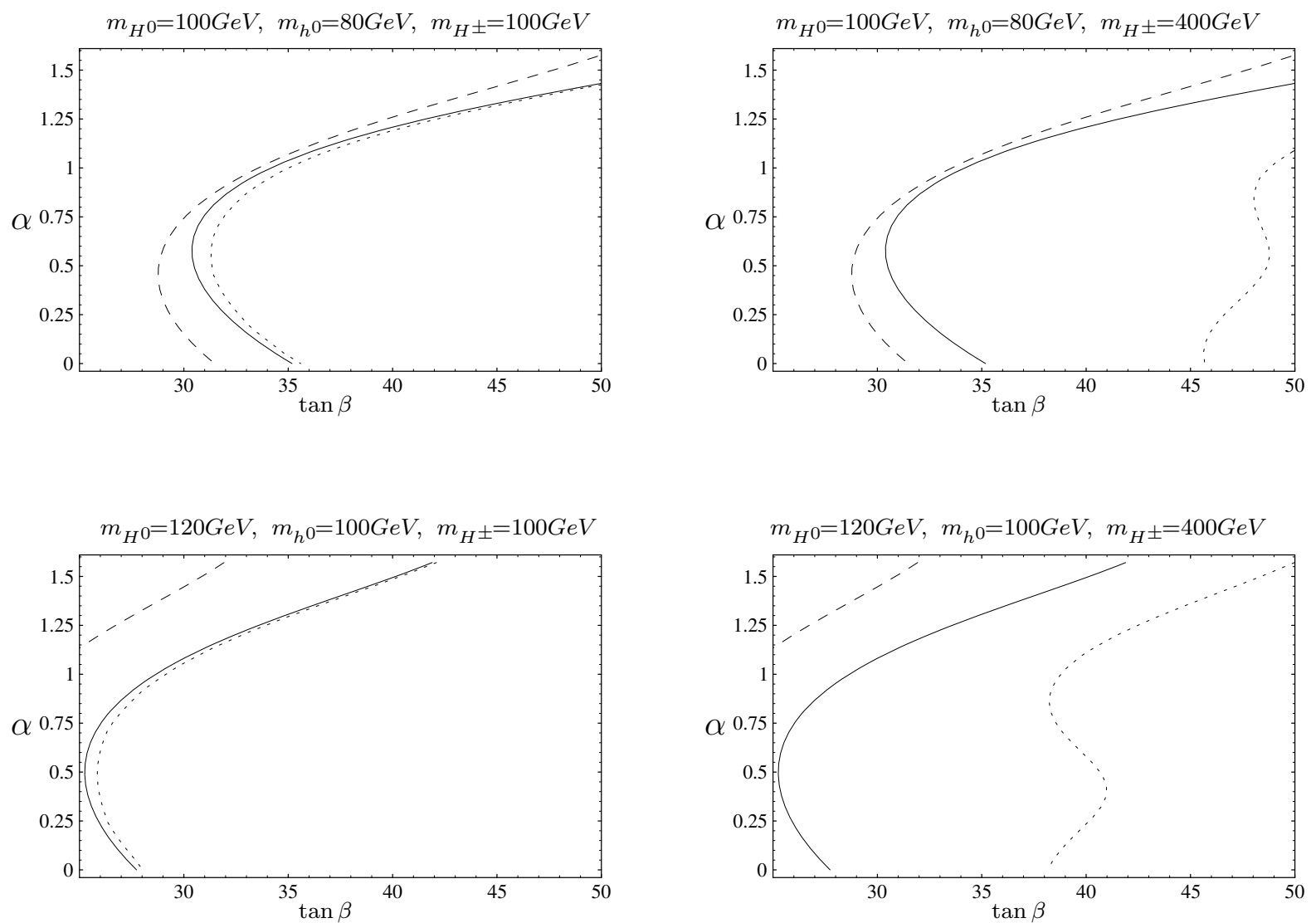

Figure 4: Allowed (shaded) areas in the $\alpha$ and $\tan \beta$ plane for $m_{A^{0}}=400 \mathrm{GeV}$ and various choices of neutral Higgs boson masses.
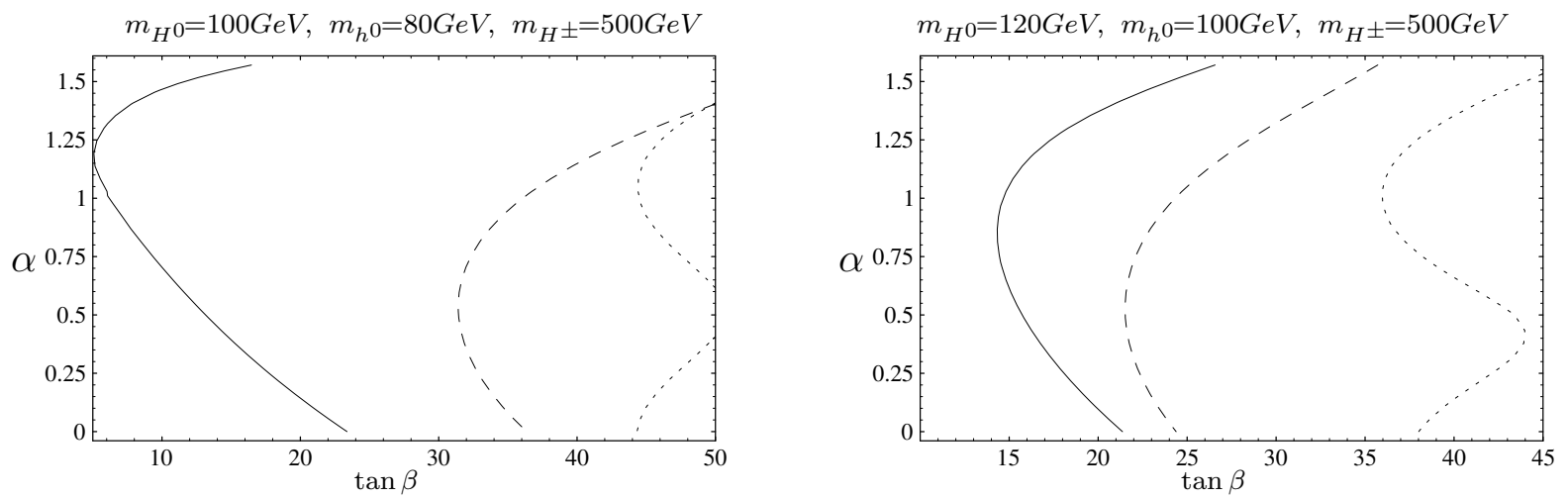

Figure 5: Allowed (shaded) areas in the $\alpha$ and $\tan \beta$ plane for $m_{A^{0}}=500 \mathrm{GeV}$ and various choices of neutral Higgs boson masses. 